\title{
Incidence des caractéristiques structurales du sous-sol sur l'entrée en production de Tuber melanosporum et d'autres champignons mycorhiziens
}

\author{
G Callot *, B Jaillard \\ avec la participation de JP Braye et M Jalade \\ Laboratoire de science du sol, Inra, 2, place Pierre-Viala, F-34060 Montpellier cedex 1, France
}

(Reçu le 28 mars 1996; accepté le 25 juin 1996)

\begin{abstract}
Résumé - Les sites de récolte d'ascocarpes de Tuber melanosporum Vitt ont été relevés durant 4 années consécutives dans une truffière de chênes verts (Quercus ilex) mycorhizés par Tuber melanosporum. Le taux de mycorhization par Tuber et d'autres champignons a été contrôlé après 11 années de plantation. Parallèlement, une étude pédologique détaillée a permis de distinguer différentes unités de sols au sein de cette truffière. L'analyse cartographique de cet ensemble de données a montré que le statut mycorhizien et les sites de récolte des champignons étaient en relation avec les caractéristiques structurales du sous-sol. La présence d'un sous-sol argileux peu perméable est défavorable aux fructifications fongiques tant épigées qu'hypogées. Les sols qui présentent un encroûtement calcaire à faible profondeur sont défavorables au développement des champignons hypogés de type Tuber, mais favorables aux champignons épigés. Les sols les plus favorables au développement de Tuber melanosporum sont des sols caractérisés par un horizon très poreux développé au contact du substratum calcaire. Cet horizon (appelé $B_{b i o}$ par les auteurs) est caractérisé par une importante activité biologique.
\end{abstract}

autoécologie / calcaire / champignon / mycorhize / sol / Tuber

Summary - Effect of structural characteristics of subsoil on the fruiting of Tuber melanosporum and other mycorrhizal fungi. The collection sites for ascocarps of Tuber melanosporum Vitt were noted over four consecutive years in a truffle bed of live oaks (Quercus ilex) mycorrhized by T melanosporum. The mycorrhizas of Tuber and other fungi were checked 11 years after planting. At the same time, a detailed soil survey made it possible to distinguish different soil units within the truffle bed. Cartographic analysis of the data set showed that the mycorrhizal status and the location of the fungus collection sites were related to the structural characteristics of the subsoil. The presence of a slightly permeable clay subsoil discourages fruiting in both epigeal and hypogeal fungi. Soils presenting a shallow calcareous crust discourage the growth of hypogeal Tuber-type fungi, but encourage that of epigeal fungi. T melanosporum grows best in soils with a very porous horizon developed in contact with the calcareous bedrock. This horizon (termed $B_{\mathrm{bio}}$ by the authors) is characterized by an intensive biological activity.

autoecology / fungi / limestone / mycorrhizas / subsoil / Tuber

* Correspondance et tirés à part 


\section{INTRODUCTION}

La production de truffe noire dite de Périgord (Tuber melanosporum Vitt) est en régression continuelle depuis le début du siècle : 1370 t en 1889, 12 t en 1990/1991 (enquête Oniflhor, 1992). Les causes de cette diminution résultent d'un ensemble de facteurs humains et technologiques : disparition des chercheurs de truffes suite aux guerres de 1914-1918 et 1939-1945, exode rural, perte du savoir-faire empirique, fermeture des truffières sauvages et anciennes truffières, mauvaise gestion des nouvelles plantations (Grente et Delmas, 1973/1974). Malgré les efforts de replantation amorcés à partir de 1970, avec des plants mycorhizés (Chevalier, 1972 ; Chevalier et Grente, 1973 ; Chevalier et al, 1973), la production continue de diminuer. Un bilan de 10 années d'observations (Chevalier et Poitou, 1988) a montré que, si, dans certaines situations, l'entrée en production de Tuber melanosporum peut intéresser plus de $80 \%$ des arbres (noisetiers) après 4 ou 5 ans de plantation, dans la plupart des cas, les arbres producteurs ne représentent que 10 à $20 \%$ des plantations. Le contrôle du taux de mycorhization des arbres dans les situations improductives révèlent que les systèmes racinaires initialement mycorhizés par Tuber melanosporum sont souvent contaminés par d'autres espèces de Tuber ou des basidiomycètes.

La truffe, champignon souterrain inféodé aux terrains calcaires, se développe dans des milieux aérés et drainants comme l'avaient déjà remarqué les auteurs anciens (de Bosredon, 1887 ; Chatin, 1892 ; Pradel, 1914 ; Malençon, 1938). Ce champignon bien adapté aux climats méditerranéens redoute toutefois une sécheresse estivale prolongée. Aussi, dans certains cas, la production a-t-elle pu être augmentée avec une irrigation modérée (LeTacon et al, 1982 ; Giraud et Verlhac, 1987), mais tout excès d'eau reste défavorable à la mycorhization de Tuber melanosporum (Olivier et Mamoun, 1989, 1990) et, d'une manière générale, au développement du champignon.

Le travail présenté ici est fondé sur une analyse détaillée des structures du sous-sol et des sites de récolte d'une truffière de truffe noire du Périgord (Tuber melanosporum), suivie régulièrement au cours des quatre premières années de production. Dans les terrains calcaires, les structures du sous-sol peuvent varier sur quelques mètres seulement, et induire d'importantes variations locales du fonctionnement hydrique du sol
(Jaillard, 1982 ; Callot et al, 1983 ; Voltz, 1986). Nous montrons que ces hétérogénéités structurales ont une incidence sur la mycorhization des arbres et le développement des champignons mycorhiziens.

\section{MATÉRIEL ET MÉTHODES}

\section{Contexte pédoclimatique}

La truffière étudiée occupe une superficie d'environ un hectare. Elle est située dans la région de Montpellier (France). Le climat est de type méditerranéen, à forte influence maritime. La température moyenne annuelle est de $14^{\circ} \mathrm{C}$, et la pluviométrie moyenne de $720 \mathrm{~mm}$ par an (410 à $1100 \mathrm{~mm}$ par an). Ce climat est caractérisé par un fort déficit hydrique estival avec des températures moyennes de $23^{\circ} 5$ en juillet.

La parcelle repose sur des calcaires lacustres du Lutétien (Tertiaire). Ces calcaires marneux blancs, riches en débris végétaux et coquilliers, présentent des alternances de sédimentation avec des niveaux plus ou moins indurés, marneux, gréseux et pisolithiques. Ce substratum calcaire est faiblement tectonisé avec des réseaux de fractures, comme tous les calcaires de cette région. Les sols développés sur cette formation sont de couleur brun jaune (10 YR 4/4) à brune (7,5 YR 4/4), caillouteux et bien structurés en surface. La teneur en cailloux est comprise entre 5 et $15 \%$. La texture de la terre fine est argileuse, avec une forte effervescence à $\mathrm{HCl} 0,5 \mathrm{~N}$. La couleur brune du sol traduit la présence d'un humus de type mull, révélateur d'une bonne activité biologique.

\section{Passé cultural de la parcelle}

Le plan de la truffière est reporté sur la figure 1. Avant son installation, le secteur nord-est (rangs 1 à 17) était planté en vigne, anciennement boisé en chênes verts. Le secteur médian (rangs 18 à 34 ) était planté en chênes verts, et le secteur sud-ouest (rangs 35 à 52) en vigne durant plus de 40 ans. En 1980, une plantation truffière a été installée après remembrement, défriche et préparation des sols, sans désinfection préalable. Des arbres de 2 ans mycorhizés par Tuber melanosporum (procédé Inra/Anvar ; Chevalier, 1988) ont été plantés selon un maillage régulier de $4 \times 5,5 \mathrm{~m}$. Les essences sont le chêne vert (Quercus ilex $L$ ) (357 plants), le chêne pubescent (Quercus pubescens Willd) (35 plants) et le noisetier (Corylus avellana $L$ ) (6 plants).

De 1980 à 1987, la truffière n'a pas été entretenue. En 1987, elle a été débroussaillée, et le sol travaillé jusqu'en 1990 par griffage à 1,5 $\mathrm{m}$ des troncs. Depuis 1991, un désherbage chimique de printemps (Roundup) a été préféré à l'entretien mécanisé. La truffière est irriguée par aspersion sur frondaison, à 


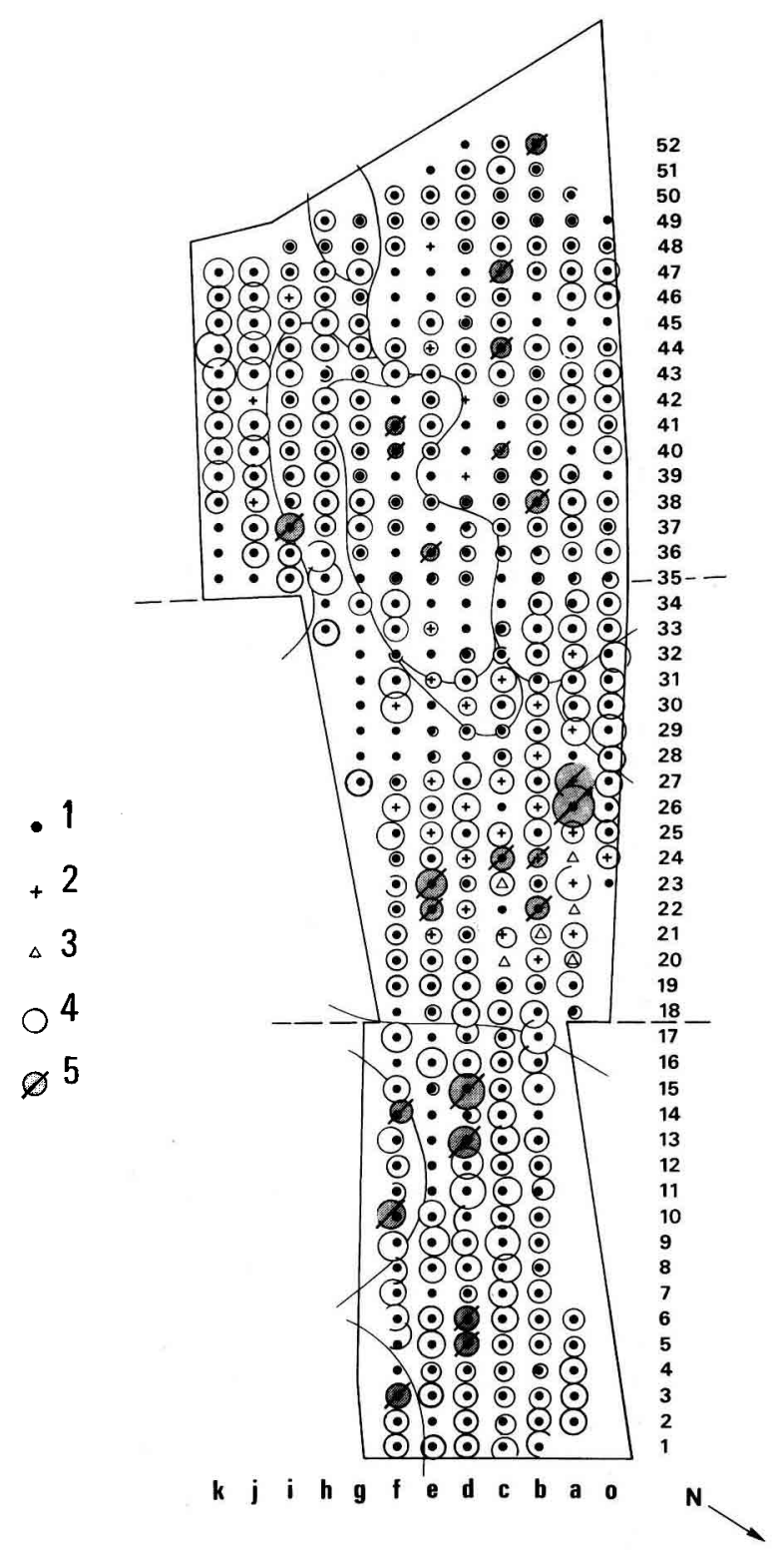

Fig 1. Plant de la truffière. Distribution spatiale et extension des brûlis après 11 années de plantation. 1 : chêne vert (Quercus ilex), 2 : chêne pubescent (Quercus pubescens), 3 : noisetier (Corylus avellana), 4 : brûlis, 5 : brûlis où le taux de mycorhization des arbres a été contrôlé.

raison de 4 à 6 apports de $20 \mathrm{~mm}$ chacun entre les mois d'avril et de septembre.

\section{Étude pédologique de la parcelle}

La cartographie des sols a été réalisée à partir de 70 sondages et de sept tranchées pédologiques. Les critères de différenciation pris en compte ont été la structure du sous-sol, la profondeur du sol et la texture de la couche de surface. Une attention particulière a été portée à la présence et à la nature des figures de recarbonation caractéristiques de phases de saturation en eau (Jaillard, 1982, 1985). L'interpolation entre les sondages a reposé sur les variations de couleur de la terre fine, la réactivité du sol à $\mathrm{HCl} 0,5 \mathrm{~N}$, et la présence de cailloux à la surface du sol. Les analyses physicochimiques ont été effectuées sur les différents horizons des sept tranchées pédologiques et sur 21 prélèvements de surface répartis sur l'ensemble de la parcelle.

Les déformations superficielles du substratum calcaire ont été mises en évidence par analyse cartographique des gradients de pente topographique. Cette méthode, généralement utilisée pour des études structurales à l'échelle régionale (Prud'homme, 1972 ; Callot, 1976), peut être appliquée à l'échelle parcellaire. L'analyse des anomalies de variations de pente des isohypses de profondeur du substratum permet de localiser les axes majeurs de fracturation.

\section{Localisation des brûlis, contrôle des mycorhizes et récolte des champignons}

Le cycle biologique de la truffe comprend deux phases bien distinctes : une phase végétative en symbiose avec la racine de l'arbre hôte (mycorhize) et une phase de fructification sans relation directe avec la racine (ascocarpe) (Montant et al, 1983 ; Giovanetti et al, 1992 ; Barry et al, 1993). La phase végétative se traduit généralement sur le terrain par la présence de brûlis, c'est-à-dire d'une zone dépourvue de végétation développée le plus souvent autour du tronc de l'arbre mycorhizé. L'absence de végétation résulte d'une action phytotoxique du mycélium de Tuber melanosporum (Fasolo Bonfante et al, 1971 ; Montacchini et Caramiello Lomagno, 1977), mais aussi d'autres champignons mycorhiziens. L'apparition d'un brûlis précède souvent l'entrée en production d'une truffière, mais tous les brûlis ne sont pas producteurs d'ascocarpes de Tuber.

En 1992, les zones d'extension des brûlis centrés auteur des arbres ont été relevés. Dans 23 sites, des racines et des mycorhizes ont été prélevées selon la méthode de Chevalier et al (1982) : prélèvements à $1 \mathrm{~m}$ du tronc de l'arbre hôte, selon quatre points cardinaux, à une profondeur comprise entre 5 et $15 \mathrm{~cm}$. Les segments de racine, de 10 à $15 \mathrm{~cm}$ de long, ont été contrôlés sous loupe binoculaire et microscope optique. Le taux de mycorhization pour chaque type de mycorhize a été évalué par une note comprise entre 1 et 5. La morphologie des racines (ramifications et couleur du cortex) a également été notée.

Les premiers ascocarpes de truffe, pour l'essentiel Tuber melanosporum, ont été récoltés au cours de l'hiver 1989/1990, soit 2 ans après la mise en culture de la truffière et 9 ans après sa plantation. La récolte des truffes, ou cavage, était effectuée à l'aide d'un chien, régulièrement et deux fois par semaine du début décembre à la mi-mars. Chaque ascocarpe récolté était pesé, et son gisement était référé sur le plan de la truffière. Relevons que la plupart des ascocarpes ont été récoltés à l'intérieur de brûlis.

En octobre 1991, une poussée de champignons a permis de localiser et d'identifier les basidiocarpes épigés de plusieurs autres espèces mycorhiziennes. 


\section{RÉSULTATS}

\section{Variabilité des sols à l'intérieur de la truffière}

L'analyse pédologique met en évidence la diversité des sols au sein de la truffière (fig 2). Une coupe géo-pédologique effectuée selon un axe NE-SW montre les différents faciès du substratum calcaire et leurs relations avec les sols (fig 3). Les différences de dureté et de fracturation de la roche amènent à distinguer deux ensembles. Le premier consiste en calcaires marneux fossilifères, assez durs et fracturés. Le second ensemble, situé au sud-ouest de la par-

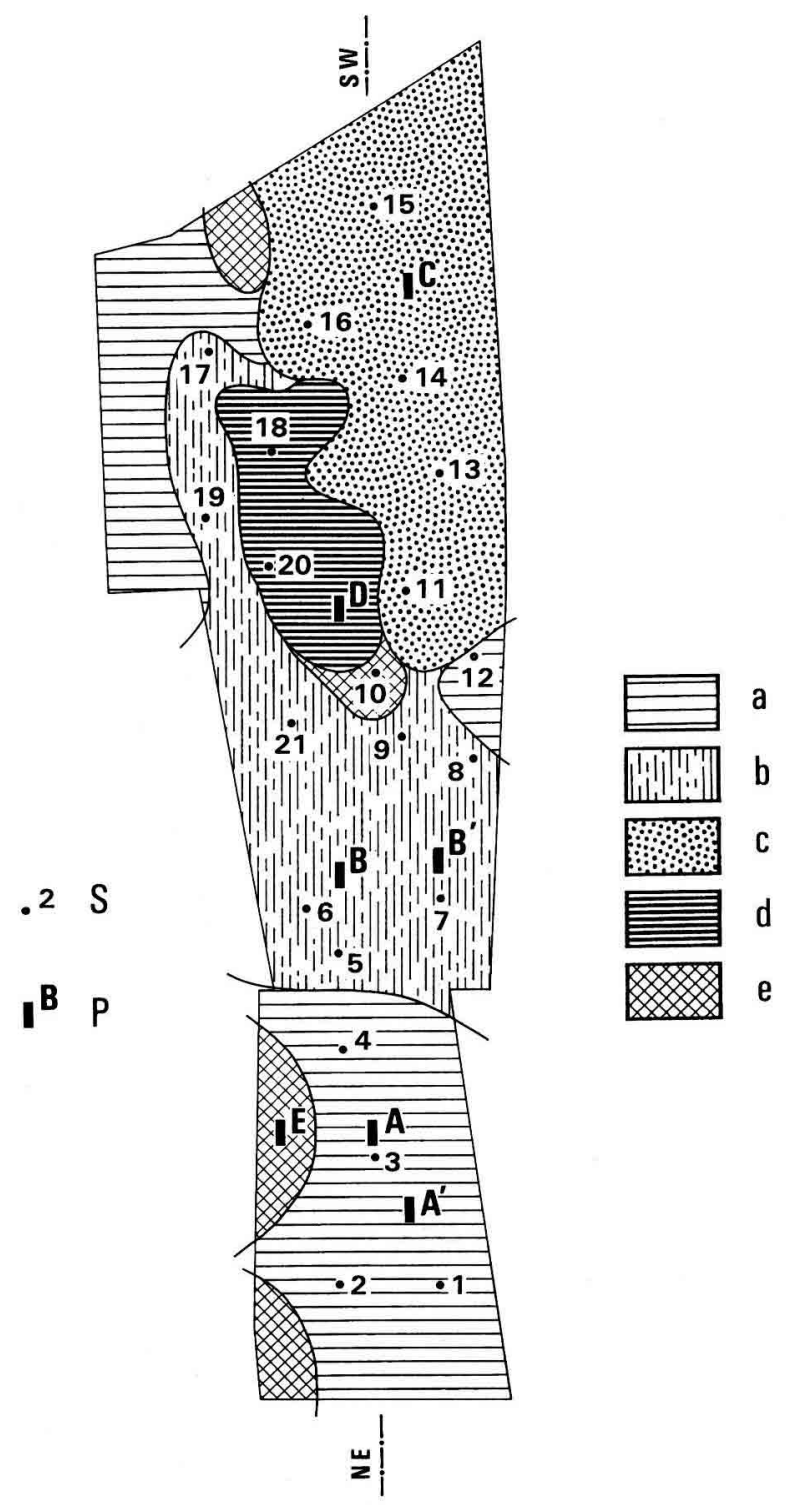

Fig 2. Carte des sols de la truffière et localisation des sondages. $a$ : RENDISOL fersiallitique, $b$ : FERSIALSOL carbonaté, $c$ : RENDOSOL à horizon pétrocalcique, $d$ : CALCOSOL, $e$ : CALCOSOL à horizon calcarique, $S$ : localisation des prélèvements de surface, $\mathrm{P}$ : profils pédologiques, NE-SW : axe de la coupe présentée sur la figure 3 . celle, consiste en calcaires plus tendres, peu ou pas fissurés, intercalés de lits marneux et de niveaux bréchiques.

Les calcaires marneux fracturés supportent deux types de sols, tous les deux caractérisés par la pénétration du matériau terrigène au sein du substratum calcaire sous forme de poches de dissolution. Les sols, notés a sur la figure 2, sont des Rendisols fersiallitiques (Afes, 1995). II s'agit de sols peu profonds (de 25 à $40 \mathrm{~cm}$ de profondeur), brun jaune (7,5 YR 4/4), graveleux, à fraction fine réagissant fortement à $\mathrm{HCl}$. La structure du sol, grumeleuse en surface, devient polyédrique sub-anguleuse en profondeur. Au contact du substratum calcaire, quelques accumulations d'aiguilles de calcite peuvent être observées par place (Callot et al, 1985). Le second type de sols, noté $b$, s'apparente à des Fersialsols carbonatés (Afes, 1995). II s'agit de sols brun rouge ( $5 \mathrm{YR} 4 / 4$ ), graveleux, à terre fine argileuse, de 40 à $80 \mathrm{~cm}$ de profondeur. Ces sols présentent un horizon de surface à structure grumeleuse, et un horizon $\mathrm{B}$ poreux à structure polyédrique. La terre fine fait modérément effervescence à $\mathrm{HCl}$. L'absence de reprécipitations calcaires dans l'horizon $B$ et la présence de langues de pénétration de l'horizon $\mathrm{B}$ dans le substratum calcaire altéré montrent que les sols de ces deux unités $a$ et $b$ sont des sols drainants.

Les calcaires marneux peu fracturés supportent trois types de sols. Les sols les plus calcaires, notés $c$ sur la figure 2 , se définissent comme des RENDOSOLS à horizon pétrocalcarique (Afes, 1995). Ce sont des sols peu profonds (de 25 à $40 \mathrm{~cm}$ de profondeur), brun jaune foncé (10 YR 4/4), caillouteux, à très forte effervescence à $\mathrm{HCl}$. La couche de surface présente une structure grumeleuse à tendance pulvérulente. À partir de $25 \mathrm{~cm}$ de profondeur, on note la présence d'un encroûtement calcaire développé dans le substratum calcaire. Cet encroûtement calcaire caractérise un horizon $\mathrm{K}_{\mathrm{m}}$ faiblement induré avec feutrage d'aiguilles de calcite.

Les sols notés $d$ sont des sols bruns calcaires ou Calcosols (Afes, 1995). II s'agit de sols de couleur brun foncé (10 YR 3/4), de texture plus argileuse et de teneur en matière organique plus importante que celle des autres sols de la parcelle. Leur terre fine est peu effervescente à $\mathrm{HCl}$. La structure des couches de surface est grumeleuse. Un horizon $B$ argileux, à structure prismatique avec faces de glissement, apparaît à partir de $40 \mathrm{~cm}$ de profondeur et repose sur le substratum calcaire à $80 \mathrm{~cm}$. Le premier décimètre de cet horizon est marqué par des reprécipitations car- 
A

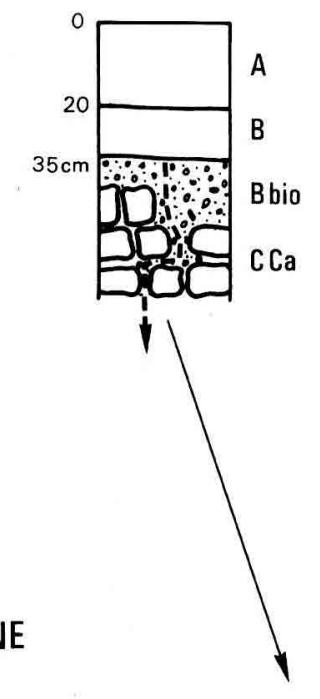

B

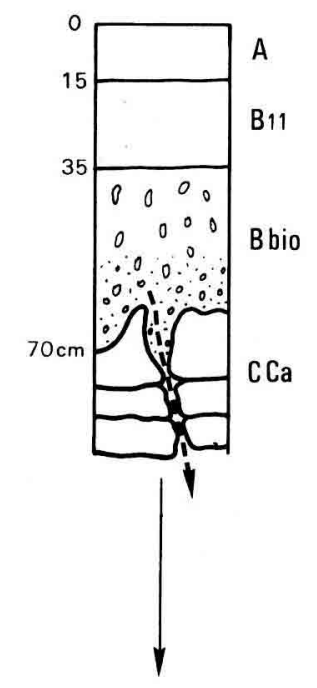

E

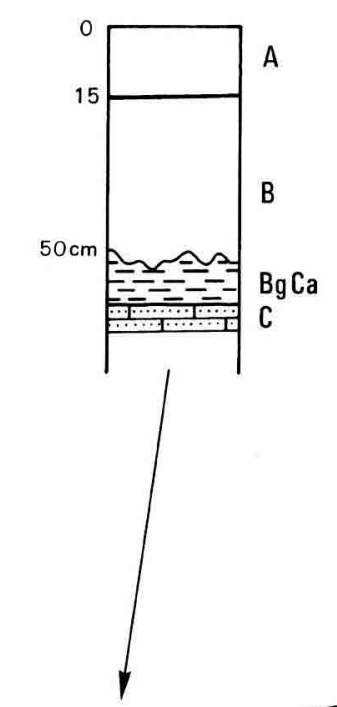

D

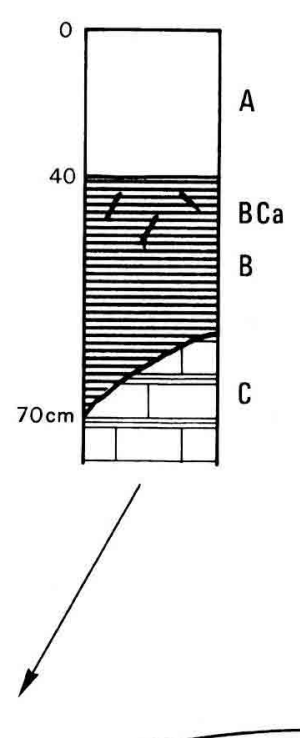

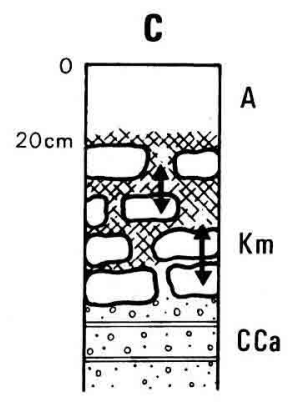

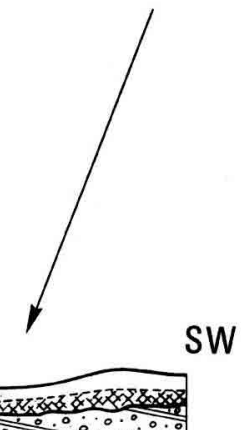

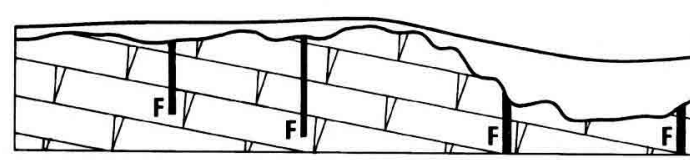

surface du
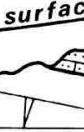

du sol

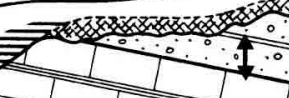

I

F: faille

HORIZONS DIAGNOSTIQUES DU SOUS-SOL

Calcaires marneux fossilifères

ㄱ avec bancs durs fissurés

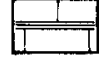
avec lits marneux

avec niveaux sableux

à niveaux gréseux bréchiques horizons poreux avec cavités et chenaux biologiques

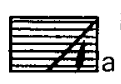

horizons argileux compacts, a) cellules de calcite

$=$ horizons ocre jaune, à calcaire pulvérulent

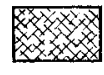

horizon à pseudomycélia calcaires (aiguilles de calcite)
INCIDENCES SUR

LE FONCTIONNEMENT HYDRIQUE

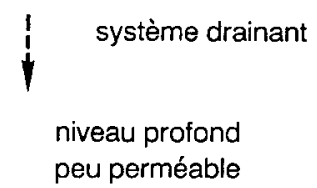

hydromorphie temporaire

alternance de phases de saturation et de dessèchement

Fig 3. Coupe géopédologique de la truffière selon l'axe NE-SW (cf fig 2).

bonatées vermiformes, identifiées comme des racines calcifiées et révélatrices d'un milieu peu perméable (Jaillard, 1985 ; Jaillard et al, 1991).

Les sols notés $e$ sont peu représentés sur la parcelle. Ils peuvent être définis comme des CALCOSOLs à horizon calcarique (AFES, 1995). Ils sont de couleur brun clair (7,5 YR 6/4), graveleux, peu caillouteux, légèrement battants, à forte effervescence à $\mathrm{HCl}$. Ils présentent à partir de $40 \mathrm{~cm}$ un niveau jaunâtre d'altération avec précipitations localisées de calcaire en nodules friables pulvérulents (horizon $\mathrm{K}$ ) associées à des taches ocres d'oxydo-réduction. Cet horizon est révélateur de phases de saturation temporaire en eau (Jaillard, 1982). Le substratum calcaire altéré est situé vers $70 \mathrm{~cm}$ de profondeur.

Finalement, l'étude pédologique de la parcelle a permis de distinguer des sols sans reprécipitation de carbonate de calcium dans l'horizon $B$ (unités $a$ et $b$ ), des sols avec encroûtement calcaire (horizon $\mathrm{B}_{\mathrm{Ca}}$ ) à faible profondeur (unités $c$ ), des sols avec nodules calcaires et taches d'oxydo-réduction (unité $e$ ) et des sols présentant un niveau argileux peut perméable (unité $d$ ). 


\section{Caractéristiques physicochimiques des sols}

Les caractéristiques physicochimiques des différentes unités de sol sont présentées sur les tableaux I et II. Elles sont compatibles avec celles de sols aptes à la culture de la truffe (Chevalier et Poitou, 1988). Des différences existent toutefois au niveau des teneurs en calcaire et des teneurs en cuivre. Les sols de l'unité $c$, qui présentent un encroûtement calcaire peu profond, sont les plus calcaires ( $56 \pm 7 \%$ de calcaire total, $14 \pm 2 \%$ de calcaire actif). Les sols de l'unité $d$ à sous-sol argileux sont les moins calcaires ( $26 \pm 2 \%$ de calcaire total et $5 \pm 1 \%$ de calcaire actif).

Les teneurs en cuivre total des couches de surface des sols de la parcelle sont comprises entre 24 et $367 \mathrm{ppm}$ (tableau II). Les plus faibles teneurs (de 24 à $88 \mathrm{ppm}$ ) sont observées dans l'unité $b$ où les sols sont profonds à bon drainage interne. Les teneurs les plus élevées (228 à $367 \mathrm{ppm}$ ) sont observées dans l'unité $c$, où les sols présentent un encroûtement calcaire $\mathrm{K}_{m}$ peu profond. Les teneurs varient entre 40 et $126 \mathrm{ppm}$ dans l'unité $a$, et entre 85 et 210 ppm dans l'unité d. Les teneurs en cuivre les plus faibles sont relevées dans le secteur médian anciennement occupé par un bois de chêne vert, où les sols sont aussi les plus drainants (unité $b$ ). Les teneurs les plus élevées s'observent dans des sols du secteur S-W autrefois planté en vigne. II semble donc que, d'une manière générale et au sein d'une même parcelle, la teneur en cuivre des couches de surface des sols est en relation avec la structure des couches du sous-sol qui règlent le drainage interne du sol. Cette relation n'a pas été expliquée.

Si des phénomènes de toxicité en cuivre existent en sol acide au-delà de 200 à 400 ppm de cuivre total (Delas, 1981), l'appréciation d'un seuil de toxicité en sol calcaire paraît plus délicate du fait de la précipitation du cuivre sous forme carbonate. D'une manière générale, la disponibilité du cuivre pour la plante diminue lorsque le $\mathrm{pH}$ augmente (Drouineau et Mazoyer, 1962). En sol calcaire, les concentrations en cuivre dans la solution du sol sont faibles, comprises entre 1 et 4 ppm (Boischot et Quillon, 1952). Elles sont toujours inférieures à $5 \mathrm{ppm}$, seuil au-delà duquel le mycélium de truffe commence à réduire sa croissance (Poitou et Cassin, 1989). Les quelques données existantes révèlent des récoltes de truffes dans des sols calcaires avec 80 à 100 ppm de Cu total (Poitou et Cassin, 1989). Un antécédent cultural vigne, malgré des teneurs en cuivre parfois élevées, est généralement favorable pour l'implantation de truffières. Toutefois les teneurs élevées constatées dans l'unité $c$ pourraient avoir contribué à la disparition des mycorhizes de Tuber melanosporum dans ce secteur de la parcelle.

\section{Répartition et identification des brûlis par unités de sol}

Tous les brûlis dans lesquels ont été récoltés au moins un ascocarpe de Tuber melanosporum au

Tableau I. Propriétés physicochimiques des sols des cinq unités identifiées au sein de la truffière (cf figs 2 et 3 ).

\begin{tabular}{|c|c|c|c|c|c|c|c|c|c|c|c|c|}
\hline \multirow{3}{*}{$\begin{array}{l}\text { Unités de sols: } \\
\text { Référence profil: } \\
\text { Profondeur }(\mathrm{cm})\end{array}$} & \multicolumn{2}{|r|}{ a } & \multicolumn{3}{|c|}{$\mathrm{b}$} & \multicolumn{2}{|r|}{ c } & \multicolumn{3}{|c|}{$d$} & \multicolumn{2}{|r|}{ e } \\
\hline & \multicolumn{2}{|c|}{$A$} & \multicolumn{3}{|c|}{$B$} & \multicolumn{2}{|r|}{$c$} & \multicolumn{3}{|c|}{$D$} & \multicolumn{2}{|c|}{$E$} \\
\hline & $0-15$ & $15-25$ & $0-5$ & $5-15$ & $15-35$ & $0-20$ & $20-25$ & $0-5$ & $5-15$ & $15-40$ & $0-15$ & $15-30$ \\
\hline Calcaire total $(\%)$ & 44 & 58 & 36 & 33 & 36 & 60 & 86 & 26 & 28 & 17 & 51 & 59 \\
\hline Calcaire actif (\%) & 10 & 12 & 6 & 4 & 5 & 17 & 21 & 5 & 5 & 1 & 13 & 16 \\
\hline Carbone $(\%)$ & 1,7 & 1,3 & 1,6 & 1,1 & 1,1 & 1,7 & 0,5 & 2,6 & 1,7 & 1,0 & 1,1 & 0,9 \\
\hline $\mathrm{C} / \mathrm{N}$ & 10,1 & 9,8 & 9,9 & 9,1 & 9,4 & 9,7 & 12,3 & 9,4 & 9,1 & 8,6 & 10,9 & 11,3 \\
\hline Argile (\%) & 30 & 29 & 23 & 25 & 25 & 24 & 20 & 32 & 33 & 35 & 26 & 26 \\
\hline $\mathrm{pH}$ & 8,1 & 8,1 & 8,0 & 8,1 & 8,4 & 8,1 & 8,4 & 7,9 & 8,0 & 8,1 & 8,3 & 8,4 \\
\hline CEC $(\mathrm{meg} / 100 \mathrm{~g})$ & 16,9 & nd & 14,4 & 14,0 & 13,3 & 11,9 & 2,4 & 21,9 & 19,9 & 21,9 & nd & nd \\
\hline K échangeable $\left(\mathrm{g} \mathrm{kg}^{-1}\right)$ & 0,10 & nd & 0,19 & 0,12 & nd & 0,20 & 0,28 & 0,35 & 0,21 & nd & nd & nd \\
\hline $\mathrm{P}_{2} \mathrm{O}_{5}$ total $\left(\mathrm{g} \mathrm{kg}^{-1}\right)$ & 0,63 & nd & 0,90 & 0,86 & 0,70 & 1,36 & 1,20 & 1,05 & 1,03 & nd & 0,8 & nd \\
\hline Cu total $\left(\mathrm{g} \mathrm{kg}^{-1}\right)$ & 114 & 57,7 & 47,8 & 44,9 & 35,1 & 309 & 36,1 & 270 & nd & 18,8 & nd & nd \\
\hline
\end{tabular}


Tableau II. Teneurs en calcaire total, en calcaire actif et en cuivre total des couches de surface des sols de la truffière.

\begin{tabular}{|c|c|c|c|c|c|}
\hline Unités de sols & $\begin{array}{l}\text { Référence } \\
\text { prélèvement }\end{array}$ & $\begin{array}{c}\text { Calcaire total } \\
(\%)\end{array}$ & $\begin{array}{c}\text { Calcaire actif } \\
(\%)\end{array}$ & $\begin{array}{l}\text { Cuivre total } \\
\qquad(p p m)\end{array}$ & $\begin{array}{l}\text { Site producteur de } \\
\text { Tuber melanosporum }\end{array}$ \\
\hline & 1 & 42 & 10 & 126 & - \\
\hline & 2 & 46 & 14 & 84 & + \\
\hline \multirow[t]{8}{*}{$a$} & 3 & 44 & 12 & 40 & + \\
\hline & 4 & 49 & 14 & 90 & + \\
\hline & $A^{\prime}$ & 44 & 10 & 114 & - \\
\hline & 12 & 40 & 10 & 60 & - \\
\hline & 5 & 35 & 10 & 26 & - \\
\hline & 6 & 27 & 7 & 24 & - \\
\hline & 7 & 19 & 4 & 58 & + \\
\hline & 8 & 30 & 6 & 67 & + \\
\hline \multirow[t]{7}{*}{$b$} & 9 & 26 & 4 & 47 & + \\
\hline & 17 & 30 & 7 & 88 & - \\
\hline & 19 & 24 & 4 & 62 & + \\
\hline & 21 & 40 & 6 & 27 & + \\
\hline & $B$ & 33 & 4 & 45 & + \\
\hline & 11 & 43 & 12 & 367 & - \\
\hline & 13 & 59 & 13 & 228 & - \\
\hline \multirow[t]{5}{*}{$c$} & 14 & 59 & 13 & 228 & - \\
\hline & 15 & 63 & 18 & 266 & - \\
\hline & 16 & 51 & 13 & 306 & - \\
\hline & C & 60 & 17 & 309 & - \\
\hline & 18 & 23 & 5 & 85 & - \\
\hline \multirow[t]{2}{*}{$d$} & 20 & 24 & 4 & 86 & - \\
\hline & $D$ & 28 & 5 & 210 & - \\
\hline \multirow[t]{2}{*}{$e$} & 10 & 26 & 6 & 60 & + \\
\hline & $E$ & 51 & 13 & nd & - \\
\hline
\end{tabular}

Les sites producteurs ou non de Tuber melanosporum ont été notés $(+)$ et $(-)$ respectivement.

cours des 4 années de récolte ont été considérés comme producteurs. La figure 1 montre que les brûlis sont bien développés sur l'ensemble de la parcelle, à l'exception toutefois du secteur S-W.
Le tableau III présente la proportion de brûlis producteurs par unité de sols. II permet de préciser que les proportions de brûlis dans les unités de sols $a, b, c$ et $e$ sont supérieures à $75 \%$, alors

Tableau III. Distribution des brûlis productifs ou non de Tuber melanosporum dans les différentes unités de sols de la truffière après les 4 premières années de production.

\begin{tabular}{|c|c|c|c|c|c|}
\hline \multirow[t]{2}{*}{ Unités de sols } & \multirow[t]{2}{*}{ Nombre d'arbres hôtes } & \multicolumn{2}{|c|}{ Brûlis centrés } & \multicolumn{2}{|c|}{ Brûlis productifs de Tuber melanosporum } \\
\hline & & Nombre & Proportion (\%) & Nombre & Proportion (\%) \\
\hline$a$ & 124 & 100 & 80 & 20 & 16 \\
\hline$b$ & 110 & 83 & 75 & 41 & 37 \\
\hline$c$ & 108 & 86 & 79 & 0 & 0 \\
\hline$d$ & 35 & 23 & 66 & 0 & 0 \\
\hline$e$ & 21 & 16 & 76 & 1 & 5 \\
\hline
\end{tabular}


qu'elle n'est que de $62 \%$ dans l'unité de sol $d$. Les brûlis producteurs se trouvent principalement dans les unités $a$ et $b$, où ils représentent respectivement 20 et $41 \%$ de l'ensemble des brûlis de chaque unité. Les unités de sols $a$ et $c$ présentent la même proportion de brûlis, mais l'unité $c$ ne contient aucun brûlis productif. Ces observations montrent qu'il existe une relation assez étroite entre les unités de sols et les brûlis productifs de Tuber melanosporum. Les phases de fructification et de développement du champignon apparaissent ainsi très dépendantes des conditions de milieu.

Le contrôle de mycorhization des systèmes racinaires, dans certains brûlis des différentes unités (tableau IV), montre que les mycorhizes de Tuber melanosporum sont fréquentes dans l'unité $b$, plus localement présentes dans les unités $a, c$ et $e$, et absentes dans l'unité $d$. Plusieurs types de mycorhizes peuvent coexister au sein d'un même brûlis, éventuellement producteur de Tuber melanosporum. Dans les unités $c$ et $d$, on relève une abondance de mycorhizes adventices de type $A D$ (spinules à angle droit) (Verlhac et al, 1990). Le développement de pourridié (Armillaria mellea (Vahl : Fr) Kummer) et de scléroderme (Scleroderma verrucosum (Bull : Pers) (Pers) a été observé dans les unités $c$ et $d$ qui présentent un encroûtement calcaire peu profond ou un sous-sol argileux peu perméable. La mycorhiza-

Tableau IV. Statut mycorhizien des arbres au niveau de leurs brûlis dans les différentes unités de sols.

\begin{tabular}{|c|c|c|c|c|c|c|c|}
\hline \multirow[t]{2}{*}{ Unité de sols } & \multirow{2}{*}{\multicolumn{2}{|c|}{$\begin{array}{l}\text { Référence } \\
\text { arbre hôte }\end{array}$}} & \multicolumn{4}{|c|}{ Statut mycorhizien } & \multirow[t]{2}{*}{ Site producteur } \\
\hline & & & Tuber melanosporum & Autres espèces & Basidiomycètes & Divers & \\
\hline \multirow{4}{*}{$a$} & d 13 & Qi & $0 / 5$ & $T$ & & $\mathrm{~g}$ & - \\
\hline & d 15 & Qi & $3 / 5$ & $\mathrm{AD}$ & & $g$ & - \\
\hline & d 6 & Qi & $1 / 5$ & Tbr/Tbo & 3 & & ++ \\
\hline & d 5 & Qi & $0 / 5$ & $\mathrm{AD} / \mathrm{Ta} / \mathrm{T}$ & 1 & $\mathrm{Ce}$ & ++ \\
\hline \multirow{8}{*}{$b$} & a 26 & Qi & $0 / 5$ & $\mathrm{AD} / \mathrm{Tr}$ & 1 & & ++ \\
\hline & a 27 & Qp & $2 / 5$ & & 1 & & ++++ \\
\hline & e 22 & Qi & $1 / 5$ & $\mathrm{AD} / \mathrm{Tbr}$ & & & ++++ \\
\hline & e 23 & Qi & $1 / 5$ & & & & ++++ \\
\hline & i 37 & Qi & $2 / 5$ & & & $\mathrm{~g}$ & ++++ \\
\hline & c 24 & Qi & $2 / 5$ & Tbr & & & + \\
\hline & b 24 & Qp & $3 / 5$ & & & & + \\
\hline & b 22 & Qi & $0 / 5$ & $\mathrm{Ta} / \mathrm{T}$ & & & - \\
\hline \multirow{5}{*}{$c$} & b 38 & Qi & $0 / 5$ & $\mathrm{AD} / \mathrm{Tbo} / \mathrm{T}$ & $\mathrm{Sc}$ & $\mathrm{Ar}$ & - \\
\hline & c 40 & $Q p$ & $0 / 5$ & $A D$ & & $\mathrm{Ar}$ & - \\
\hline & c 44 & Qi & $2 / 5$ & $\mathrm{AD} / \mathrm{Ta}$ & & $\mathrm{Ar}$ & - \\
\hline & c 47 & $\mathrm{Qp}$ & $0 / 5$ & $\mathrm{AD} / \mathrm{Ta} / \mathrm{T}$ & & $\mathrm{Ar}$ & - \\
\hline & b 52 & Qi & $0 / 5$ & $\mathrm{AD}$ & & $\mathrm{Ar}$ & - \\
\hline \multirow{3}{*}{$d$} & $f 40$ & Qi & $0 / 5$ & $A D$ & 1 & id & - \\
\hline & f 41 & Qi & $0 / 5$ & $\mathrm{AD}$ & & $\mathrm{Ar}$ & - \\
\hline & e 36 & Qi & $0 / 5$ & $\mathrm{AD} / \mathrm{Ta}$ & Sc & $\mathrm{Ar}$ & - \\
\hline \multirow{3}{*}{$e$} & f 3 & Qi & $0 / 5$ & $\mathrm{AD} / \mathrm{Tbo} / \mathrm{T}$ & 2 & $\mathrm{~g}$ & - \\
\hline & f 10 & Qi & $2 / 5$ & Tbo & & $\mathrm{g}$ & - \\
\hline & $f 14$ & Qi & $0 / 5$ & $\mathrm{~T}$ & & id & - \\
\hline
\end{tabular}

La production d'ascocarpes de Tuber melanosporum par les arbres est également relevée. La référence du prélèvement comprend sa localisation (cf fig 1) et la nature de l'arbre hôte, avec Qi pour Quercus ilex et Qp pour Quercus pubescent. T : type Tuber sp ; Tbr : type Tuber brumale Pico ; Tbo : type Tuber borschii Vitt ; Ta : type Tuber aestivum Vitt ; Tr : type Tuber rufum Pico ; AD : type AD (spinule à angle droit) ; Sc : Scleroderma verrucosum (Bull: Pers) Pers ; Ar : Armillaria mellea (Vahl:Fr) Kummer ; Ce : Cenococcum sp ; g : présence de glomérules (probablement de Tuber) ; id : indéterminé ; (-) production nulle ; (+) production en 1992/1993; (++) production en 1991/1992 et 1992/1993; (++++) production chaque année. 
tion par Tuber melanosporum a été conservée de manière préférentielle dans les sols de l'unité $b$, où les sols sont profonds, drainants et capables de stocker l'eau dans les couches profondes. C'est aussi dans cette unité que les récoltes de truffes sont les plus importantes (fig 4).

Dans les brûlis producteurs, les racines de l'arbre hôte sont généralement tortueuses, allongées, peu ramifiées, avec un cylindre central peu développé par rapport au cortex. Les racines de ce type sont fréquentes dans les bonnes truffières, et ont été appelées "racines à stromas" par Pargney et Jalade (1995). Elles présentent

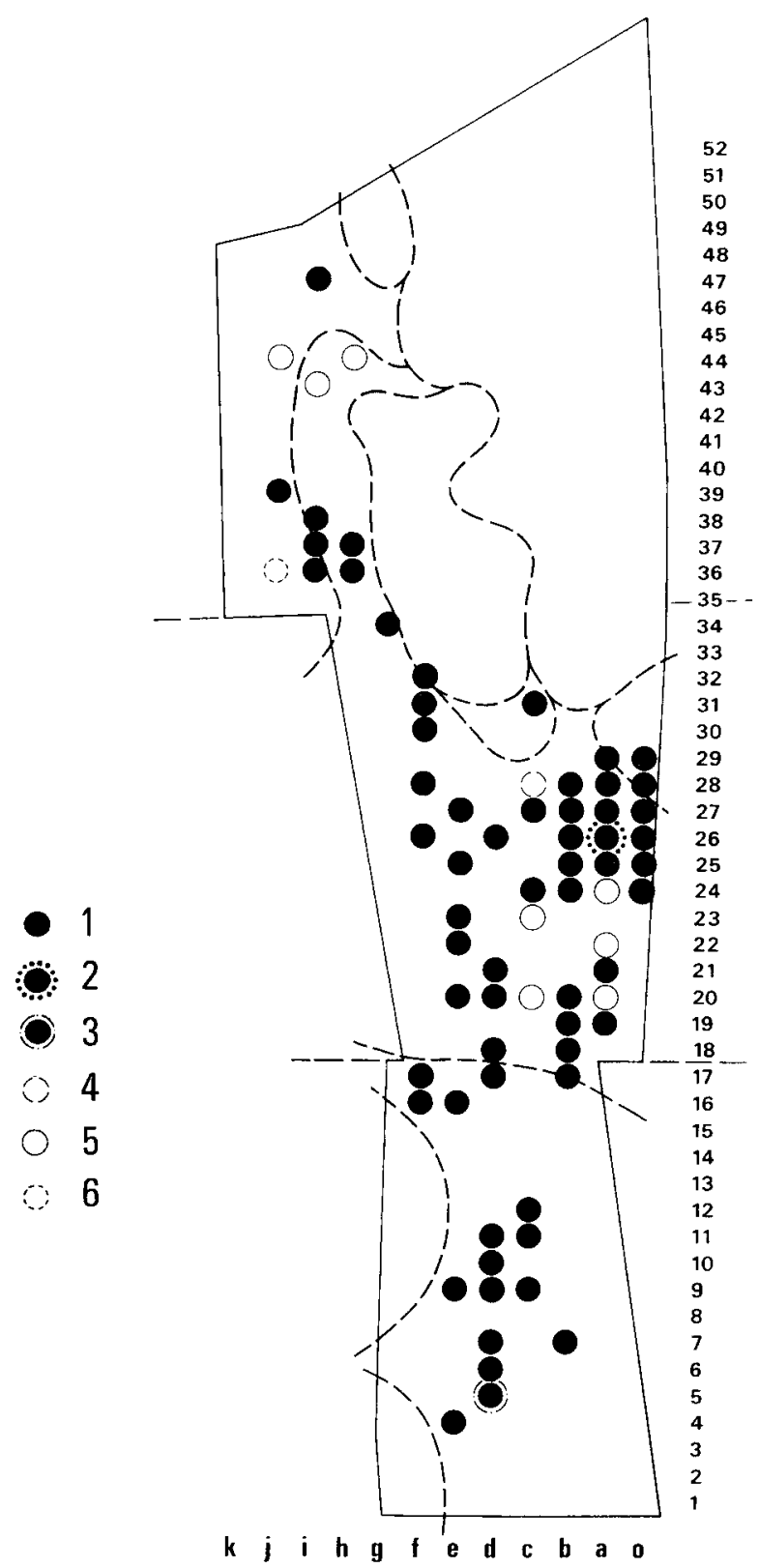

Fig 4. Localisation des brûlis productifs d'ascocarpes de Tuber au cours des 4 premières années de production. 1 : Tuber melanosporum; 2 : Tuber melanosporum et Tuber albidum ; 3 : Tuber melanosporum et Tuber brumale ; 4 : Tuber aestivum ; 5 : Tuber rufum ; 6 : Tuber malençoni. un cortex brun foncé à noir, très altéré, avec des cicatrisations d'anciennes mycorhizes. Les études en microscopie à transmission ont montré que les cellules corticales de ces «racines à stromas" étaient riches en composés phénoliques (Pargney et Brimont, 1995). Relevons qu'un système racinaire composé de telles racines et dépourvues de radicelles fines et actives semble peu absorbant. Cette morphologie pourrait expliquer pourquoi nombre d'arbres truffiers présentent un aspect chétif. Dans l'unité $c$, où les arbres sont bien développés, le système racinaire est au contraire très ramifié, avec de nombreuses radicelles non mycorhizées par Tuber melanosporum.

\section{Morphologie comparée de profils pédologiques dans des sites producteurs ou non de Tuber melanosporum}

Afin de préciser la relation entre type de sols et production de Tuber melanosporum, nous avons déterminé les principaux axes de fracturation et de drainage préférentiel du substratum calcaire (fig 5). II apparaît que, dans l'unité $b$, les anomalies de gradient de pente des isohypses sont plus marquées que dans l'unité $a$. Dans l'unité $b$, le substratum apparaît ainsi plus fracturé, avec des sols plus profonds. Relevons d'ailleurs que cette unité est située en position dépressionnaire, et que l'axe de cette dépression constitue l'axe NWSE de fracturation principale.

L'analyse structurale des profils de sol situés au voisinage des axes de fracturation a été comparée à celle des sols situés sur substratum non fracturé (fig 5). Les profils développés au voisinage des axes de fracturation (profils de type $A$ et $B$, fig 5) montrent une transition graduelle entre le sol et le substratum avec des poches de dissolution. Au contact du substratum calcaire, on note la présence d'un horizon macroporeux, d'origine racinaire et faunique, noté $\left(\mathrm{B}_{b i o}\right)$. Cet horizon macroporeux développé au contact du substratum fracturé assure le bon drainage interne du sol. En revanche, dans les sols développés sur substratum non fracturé (profils de type $A^{\prime}$ et $B^{\prime}$, fig 5), le contact entre le sol et le substratum est toujours brutal. Dans des milieux comparables, Blonde et al (1986) ont montré que, sur substratum fracturé, les sols présentent toujours un meilleur drainage libre tout en conservant un horizon médian (B) plus humide, encadré par des niveaux plus secs. Sur substratum non fracturé, en revanche, les sols présentent un horizon de 
surface qui reste plus humide, et un horizon profond plus sec en période de ressuyage. Dans les sites producteurs de Tuber melanosporum situés au voisinage des axes de fracturation (profils de type $A$ et $B$, fig 5), nous avons effectivement constaté un ressuyage plus rapide des couches de surface après des périodes de très fortes pluies. En revanche, dans les sites non producteurs situés sur substratum non fracturé (profils de type $A^{\prime}$ et $B^{\prime}$, fig 5 ), les couches de surface restent plus humides plus longtemps après un épisode pluvieux. Si l'on resitue les profils $A^{\prime}$

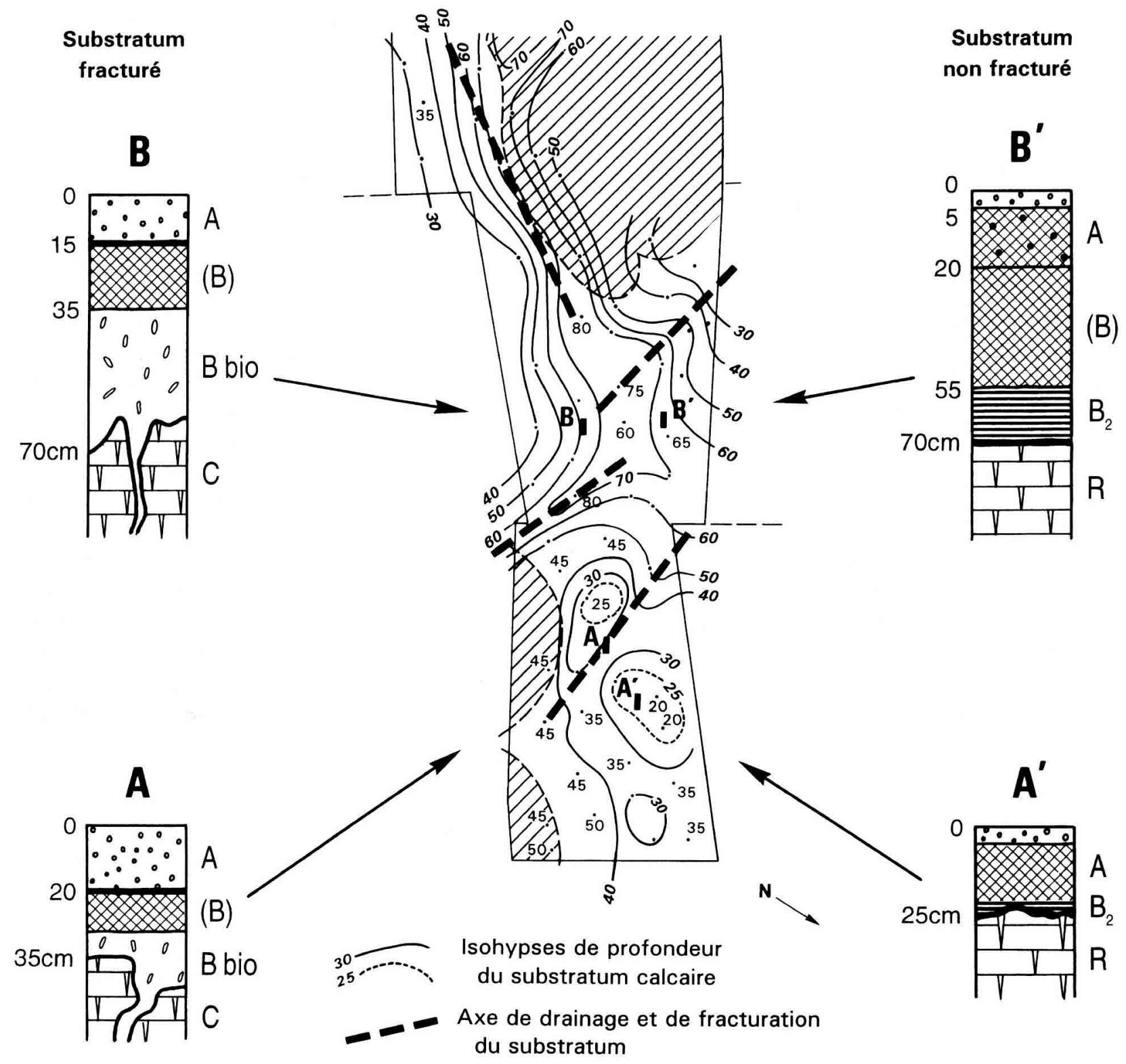

\section{Structure des couches du sol}

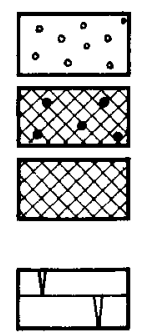

microagrégée

grumeleuse

polyédrique stable

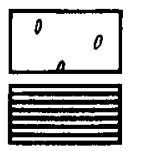

poreuse (cavités et chenaux)

compacte (argile)

Fig 5. Carte des isohypses de profondeur du substratum calcaire dans les unités $a$ et $b$ et morphologie comparée des sites productifs sur substratum fracturé (profils $A$ et $B$ ) et site improductifs sur substratum non fracturé (profils $A^{\prime}$ et $B^{\prime}$ ). 
(localisé en c8-c9, fig 1) et B' (localisé en b22b23, fig 1) sur la carte des brûlis, on constate que, près du site $A^{\prime}$, le sol est peu profond, l'enracinement est superficiel et les brûlis sont bien développés, tandis que, près du site $\mathrm{B}^{\prime}$, le sol est profond, l'enracinement est également plus profond et les brûlis sont peu marqués.

\section{Distribution des fructifications fongiques et relation avec les unités de sols}

Le relevé des fructifications des espèces fongiques récoltées, au cours de l'automne 1991 est présenté dans le tableau $V$. Les unités $d$ et $e$ présentent très peu de fructifications fongiques. L'unité $c$ est riche en carpophores de basidiomycètes, mais aucun ascocarpe de Tuber n'y a été récolté. Les unités $a$ et $b$ sont riches tant en basidiocarpes qu'en ascocarpes. Toutefois, l'unité $b$ s'avère plus productive en ascocarpes du genre Tuber et plus pauvre en basidiocarpes que l'unité $a$.

Certains brûlis productifs de Tuber melanosporum peuvent aussi être producteurs d'autres espèces de Tuber. Dans les sols de l'unité $b$ du secteur médian, les sites producteurs de Tuber rufum sont relevés uniquement autour des noisetiers. Les brûlis producteurs de Tuber ne portent pas ou peu de fructifications de Basidiomycètes, si ce n'est quelques Inocybe (espèce appelée le "truffier"). Ces observations montrent que, sous un même type de couvert végétal, les fructifications fongiques tant épigées qu'hypogées sont fortement dépendantes des conditions du sol.

\section{Évolution de la production d'ascocarpes et de la distribution spatiale des sites producteurs de Tuber melanosporum}

Les ascocarpes de Tuber melanosporum ont tous été récoltés à l'intérieur de brûlis, c'est-àdire à moins de $1,50 \mathrm{~m}$ des troncs. Cette localisation nous permet d'associer chaque site productif à un arbre producteur. L'évolution du nombre d'arbres producteurs et d'ascocarpes de Tuber melanosporum récoltés dans la truffière est présentée dans le tableau VI. II apparaît que la récolte a augmenté au cours des 4 premières années de production. Cette augmentation résulte à la fois de l'augmentation du nombre de brûlis producteurs et du nombre d'ascocarpes récoltés. En 1992/1993, la production a toutefois diminué dans l'unité $a$ et augmenté dans l'unité $b$. Les sols de l'unité $a$, peu profonds, irrigués de la même manière que ceux de l'unité $b$, ont probablement été soumis à des phases de saturation prolongée en eau. La diminution de production de truffes avec l'irrigation a souvent été constatée sur sols peu profonds (Sourzat et al, 1993). Le poids moyen des ascocarpes récoltés (de

Tableau V. Abondance des fructifications fongiques épigées et hypogées dans les unités de sol de la truffière à l'automne 1992.

\begin{tabular}{rccccc} 
Unité de sols : & $\mathrm{a}$ & $\mathrm{b}$ & $\mathrm{c}$ & $\mathrm{d}$ & $\mathrm{e}$ \\
\cline { 2 - 6 } Nombre d'arbres: & 124 & 110 & 108 & 35 & 21
\end{tabular}

Basidiomycètes

Inocybe jurana (Patouillard) Saccardo

Inocybe sp

Xerocomus rubellus Quèlet

Gyroporus castaneus (Bull:Fr) Quèlet

Cortinarius sp

Pisolithus arrhizus (Scop) S Rauschrt

Scleroderma verrucosum (Bull:Pers) Pers

Astraeus hygrometricus (Pers:Pers) Morgan

$\begin{array}{ccc}9 & 10 \\ 38 & 5 & \\ 6 & 5 & \\ 2 & - & \\ - & - & 217 \\ 16 & 7 & 24 \\ 6 & 4 & 23 \\ 3 & - & \end{array}$

$\begin{array}{ccc}7 & - & 11 \\ 1 & - & 1 \\ 17 & 2 & - \\ - & - & - \\ 21 & - & 1 \\ 24 & - & 3 \\ 23 & 6 & 1 \\ - & - & -\end{array}$

Ascomycètes

Morchella sp

Tuber melanosporum Vitt

Tuber borschii Vitt

Tuber aestivum Vitt

Tuber rufum Pico

$\begin{array}{lll}- & - & - \\ - & - & 1 \\ - & - & - \\ - & - & - \\ - & - & -\end{array}$


Tableau VI. Nombre de brûlis producteurs de Tuber melanosporum, nombre d'ascocarpes récoltés et poids moyen des ascocarpes au cours des 4 premières années de production dans les unités a et $b$.

Hiver:

$$
\text { Total }
$$$$
\text { Total }
$$

89/90 90/91 91/92 92/93
Unité a

$\begin{array}{llll}89 / 90 & 90 / 91 & 91 / 92 \quad 92 / 93\end{array}$
Unité b
Nombre d'arbres hôtes Nombre de brûlis producteurs Nombre d'ascocarpes récoltés Poids moyen des ascocarpes (g)

$398 \quad 398 \quad 398$

$15-18$

$\begin{array}{lll}15 & 18 & 32 \\ 47 & 57 & 135\end{array}$

$\begin{array}{lll}47 & 57 & 135\end{array}$

g) $110 \quad 80$

$\begin{array}{ccccccccc}398 & 124 & 124 & 124 & 124 & 110 & 110 & 110 & 110 \\ 41 & 5 & 6 & 13 & 9 & 10 & 12 & 19 & 32 \\ 161 & 11 & 22 & 47 & 26 & 36 & 35 & 88 & 135 \\ 87 & 140 & 95 & 104 & 57 & 81 & 64 & 74 & 118\end{array}$

l'ordre de $100 \mathrm{~g}$ ) a peu varié d'une année sur l'autre. II apparaît relativement élevé par rapport au poids moyen (20 à $50 \mathrm{~g}$ ) d'ascocarpes récolté sur des truffières naturelles. L'irrigation et la présence d'un sol à texture fine argileuse, à bonne capacité de rétention en eau, pourraient en être la cause.

L'évolution de la distribution spatiale des sites producteurs de Tuber melanosporum année après année est présentée sur la figure 6. II apparaît que les arbres premiers producteurs sont généralement localisés le long de directions préférentielles, qui correspondent approximativement aux axes de fracturation. Les brûlis bons producteurs, c'est-à-dire les brûlis où l'on récolte chaque année plus de 4 ascocarpes et où le poids annuel de récolte est supérieur à $0,5 \mathrm{~kg}$, se situent au voisinage de ces axes de production. Le suivi régulier de la production d'ascocarpes a permis d'établir une carte de fréquence de production des différents arbres de la truffière (fig 7). Cette carte met bien en évidence le fait que la

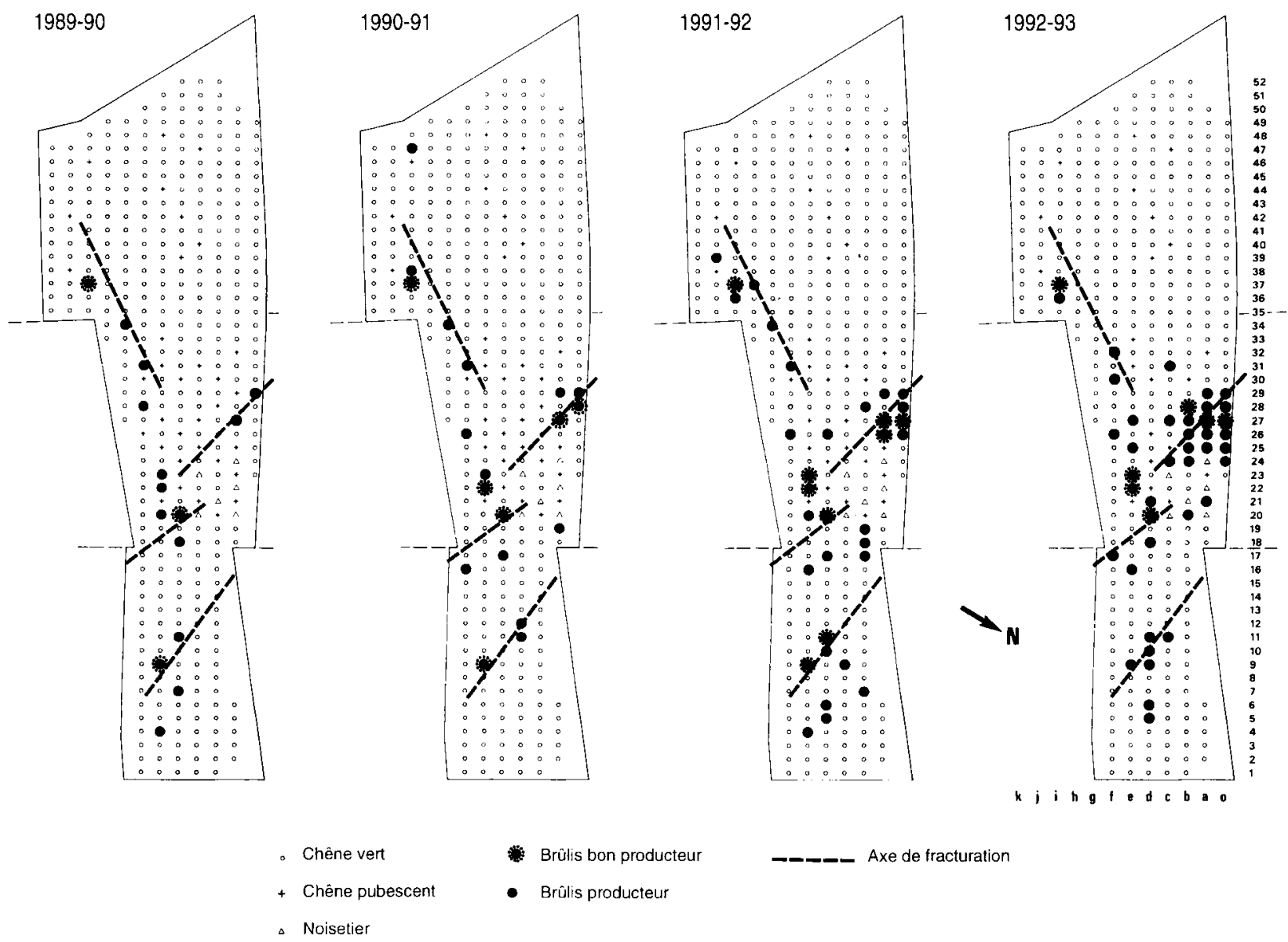

Fig 6. Localisation des sites producteurs de Tuber melanosporum année par année, et localisation des principaux axes de fracturation du substratum calcaire. 


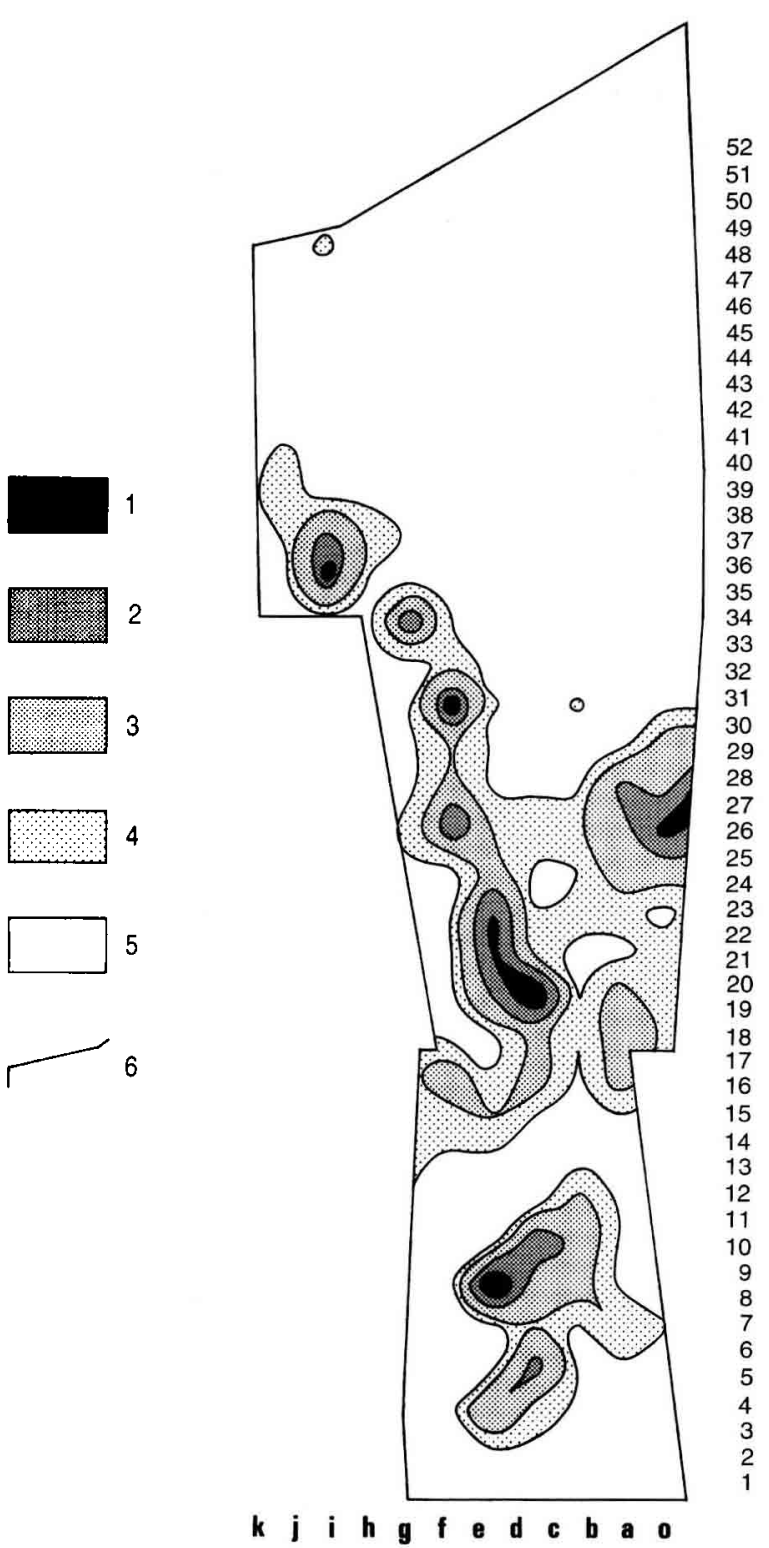

Fig 7. Carte de fréquence de production de Tuber melanosporum au cours des 4 premières années de production. 1 : zone de fréquence $4 / 4 ; 2:$ zone de fréquence $3 / 4 ; 3:$ zone de fréquence $2 / 4 ; 4:$ zone de fréquence $1 / 4 ; 5:$ zone de fréquence $0 / 4 ; 6$ : limites de la truffière.

production s'organise autour des arbres qui produisent régulièrement année après année. $\mathrm{Ce}$ résultat confirme les observations de Pradel (1914) qui écrivait que «le principal (arbre) producteur est toujours celui qui a été le point de départ de la truffière". Dans la truffière étudiée, cinq foyers primaires de production peuvent ainsi être identifiés.

\section{DISCUSSION ET CONCLUSION}

L'analyse pédologique et cartographique de la truffière étudiée montre en premier lieu que, en milieu calcaire, les sols d'une même parcelle peuvent s'avérer très hétérogènes malgré une relative homogénéité des analyses de terre des couches de surface. Cette hétérogénéité résulte de variations pétrographiques et structurales du substratum calcaire dont les conséquences sur le fonctionnement hydrique des sols sont importantes. Les sols les plus favorables au développement de Tuber melanosporum apparaissent comme des sols présentant un sous-sol fracturé et filtrant, caractérisés par la présence d'un horizon $\left(\mathrm{B}_{\mathrm{bio}}\right)$ à macroporosité biologique et fissurale, développé au contact du substratum calcaire. Les axes de fracturation du substratum calcaire constituent souvent des axes de drainage préférentiels le long desquels apparaissent les premiers brûlis producteurs de Tuber melanosporum. Dans le litho-système analysé, la présence d'un sous-sol argileux peu perméable et/ou d'un encroûtement calcaire à faible profondeur apparaissent peu favorables au développement de l'ascocarpe de Tuber melanosporum. II est clair que cette relation entre occurrence des ascocarpes de Tuber et structures du sous-sol résulte du mode de fructification hypogée des Tuber. En effet, les ascocarpes de Tuber melanosporum se développent d'avril à décembre, sous terre, et tout excès d'eau prolongé dans la couche de surface du sol, lié à la présence d'un sous-sol peu perméable, risque d'affecter leur croissance, voire d'entraîner leur pourriture. Par ailleurs, les sols qui ne présentent par un bon drainage interne ont de faibles capacités de stockage des eaux en profondeur, et donc de faibles possibilités de restitution de l'eau en période estivale.

Les caractéristiques structurales du sous-sol apparaissent ainsi déterminantes pour apprécier l'aptitude d'un sol à la trufficulture ; aussi doiventelles compléter impérativement la simple analyse de terre. De même, les aptitudes du sous-sol à évacuer les excès d'eau, mais aussi à stocker l'eau, constituent des éléments diagnostics à prendre en compte pour régler l'irrigation d'une truffière. Des doses d'irrigation excessives sur des sols peu profonds et peu drainants peuvent entraîner l'arrêt d'une production. Des études rigoureuses de fonctionnement hydrique dans les différents écosystèmes truffiers apparaissent ainsi indispensables, pour éviter des extrapolations trop rapides dans des milieux différents.

L'évolution de la production d'ascocarpes depuis l'entrée en production de la truffière montre que, si le sol est un facteur déterminant, d'autres facteurs interviennent. En effet, si la proportion de brûlis producteurs augmente d'année 
en année, l'extension de la production se fait généralement à partir de sites bon producteurs. Cette évolution pourrait être liée aux modifications induites par l'activité de la faune du sol, dont l'effet sur la structure du sol autour de l'ascocarpe a déjà été souligné (Callot et Guyon, 1990). Le suivi régulier des sites producteurs est apparu riche d'informations. Le trufficulteur aura donc intérêt à noter avec rigueur les sites de production des truffes, au cours des premières années de récolte. La distribution et l'évolution de ces sites de production renseignent sur les secteurs de la truffière qu'il faudra gérer différemment, avec des techniques adaptées (irrigation, travail du sol, mulching, enherbement, etc...). L'identification des arbres bon producteurs permettra aussi, lorsque la truffière sera plus âgée, de conserver ces bons producteurs et de supprimer les arbres situés dans des sites non ou peu productifs.

Le suivi de la production sur de plus longues périodes, dans d'autres truffières-pilotes, avec contrôles hydriques et thermiques en continu, nous permettra de préciser le fonctionnement de cet écosystème complexe, en particulier le rôle fondamental de la faune du sol.

\section{REMERCIEMENTS}

Ces recherches s'inscrivent dans un programme Agriculture-Recherche signé entre I'Inra et la région Languedoc-Roussillon, suite à la relance de la trufficulture menée par la chambre d'agriculture et le syndicat des trufficulteurs de l'Hérault. Nous tenons à remercier ici $\mathrm{C}$ Lecot, ingénieur de recherche au laboratoire de botanique de l'Ensam, pour ses déterminations mycologiques, et $\mathrm{F}$ Mazzella et $\mathrm{J}$ Cornet pour leur travail graphique. Nous tenons également à remercier les lecteurs anonymes dont les remarques et critiques ont contribué à améliorer ce manuscrit.

\section{RÉFÉRENCES}

Afes (1995) Référentiel pédologique (D Baize, MC Girard, eds), Inra, Paris, $332 p$

Barry D, Callot G, Janex-Favre MC, Parguey-Leduc A, Pargney JC (1993) Morphologie et structure des hyphes externes observées sur le péridium des Tuber à écailles : évolution au cours du développement de l'ascocarpe. Can J Bot 71, 609-619

Blonde JL, Gaiffe M, Contini D, Bruckert S (1986) Relations entre les caractères humifères des sols des plateaux jurassiens et le régime hydrique induit par la perméabilité des bancs calcaires. Pédologie $36,155-177$
Boischot P, Quillon P (1952) Fixation du cuivre dans les sols calcaires. Ann Agron 3, 359-363

Callot G (1976) Analyse d'un système géo-pédologique régional. Thèse d'État, USTL, Montpellier, $105 p$ et annexes

Callot G, Chamayou H, Maertens C, Salsac L (1983) Mieux comprendre les interactions sol-racine. Incidence sur la nutrition minérale. Inra Éditions, Paris, $325 \mathrm{p}$

Callot G, Guyon A, Mousain D (1985) Inter-relations entre aiguilles de calcite et hyphes mycéliens. agronomie 5, 209-216

Callot G, Guyon A (1990) Microstructural analysis of the truffle ascocarp, interface during development. In : Proceedings of 14th International Congress of Soil Sciences, Kyoto, Japan, 256-261

Chatin A (1892) La truffe. Botanique de la truffe et des plantes truffières. Baillère, Paris, $202 p$

Chevalier G (1972) Obtention de cultures de mycélium de truffe à partir du carpophore et des mycorhizes. C R Acad Agric 12, 981-989

Chevalier G, Grente J (1973) Propagation de la mycorhization par la truffe à partir de racines excisées et de plantules inséminatrices. Ann Phytopatho/ 5, 317

Chevalier G, Grente J, Pollacsek A (1973) Obtention de mycorhizes de différents Tuber par synthèse à partir de spores en conditions gnotoxéniques et à partir de cultures pures de mycélium en conditions axéniques et gnotoxéniques. Ann Phytopathol 5, 107-108

Chevalier G (1988) Le plant mycorhizé Inra-Anvar : un outil pour la relance de la trufficulture. Cahiers techniques INRA 19, 23-30

Chevalier G, Poitou N (1988) Facteurs conditionnant l'utilisation optimale des plants mycorhizés artificiellement par la truffe. In : Atti del 20 Congresso Internazionle sul Tartufo, Spoleto, Comunita Montana dei Monti Martani e del Serano, 409-413

Chevalier G, Giraud M, Bardet MC (1982) Interactions entre les mycorhizes de Tuber melanosporum et celles d'autres champignons ectomycorhiziens en sols favorables à la truffe. Les mycorhizes : biologie et utilisation, Dijon (5-6 mai 1982). Inra Pub, Les Colloques de l'Inra 13, 313-321

De Bosredon A (1887) Manuel du trufficulteur. Laporte, Périgueux, $130 p$

Delas J (1981) Les oligoéléments et la vigne. Viti-technique 4-6

Drouineau G, Mazoyer R (1962) Contribution à l'étude de la toxicité du cuivre dans les sols. Ann Agron 13, $31-53$

Fasolo Bonfante P, Fontana A, Montacchini F (1971) Studi sull'écologia del Tuber melanosporum. I. Dimostrazione di un effetto fitotossico. Allionia 17, 47-54

Giovannetti G, Zanini E, Paleta A (1992) Valutazioni morfofisiologiche e pedochimique di una possible fase saprofitica di Tuber spp. Micologia $e$ Vegetazione mediterranea, 81-94 
Giraud M, Verlhac A (1987) Expérimentation, valorisation des truffières. In : CTIFL, Bull FNPT, Paris, 9, $15-28$

Grente J, Delmas J (1973-1974) Perspectives pour une trufficulture moderne. Plaquette distribuée par la station de pathologie végétale de l'Inra de Clermont-Ferrand, $65 \mathrm{p}$

Jaillard B (1982) Relation entre dynamique de l'eau et organisation morphologique d'un sol calcaire. Sci Sol 20, 31-52

Jaillard B (1985) Activité racinaire et rhizostructures en milieu carbonaté. Pédologie 35, 297-313

Jaillard B, Guyon A, Maurin F (1991) Structure and composition of calcified roots, and their identification in calcareous soils. Geoderma 50, 197-210

Le Tacon F, Delmas J, Gleyze R, Bouchard D (1982) Influence du régime hydrique du sol et de la fertilisation sur la fructification de la truffe noire du Périgord (Tuber melanosporum Vitt) dans le sud-est de la France. Acta Oecologica, Oecologica applicata 3/4, 291-306

Malençon MG (1938) Les truffes européennes. Revue mycologique, Paris, hors-série, $92 \mathrm{p}$

Montacchini F, Caramiello Lomagno R (1977) Studi sull'écologia del Tuber melanosporum. II. Azione inhibitrice su specie erbacee della flora spontanea. Allionia 22, 81-85

Montant C, Kulifaj M, Gleize R (1983) Note sur la récolte de jeunes ascocarpes du Tuber melanosporum Vitt (truffe noire du Périgord) et leur évolution. C R Acad Sci 3, 296, 463-468

Olivier JM, Mamoun M (1989) Dynamique des populations fongiques et bactériennes de la rhizosphère des noisetiers truffiers. 1. Relation avec le statut hydrique du sol. agronomie 8, 711-717

Olivier JM, Mamoun M (1990) Dynamique des populations fungiques et bactériennes de la rhizosphère des noisetiers truffiers. 3 . Effet du régime hydrique sur la mycorhization et la microflore associée. agronomie $10,77-84$

ONIFLHOR (1992) Bilan de la filière truffe en France. Étude réalisée par M Courvoisier (Bio Markets Research). Oniflhor, Paris, $118 p$

Pargney JC, Brimont A (1995) Production of concentrated polyphenols by the root cap cells of Corylus associated with Tuber $_{1}$ : Ultrastructure study and element localization using electron energy loss spectroscopy and imaging. Trees 9, 149-157

Pargney JC, Jalade M (1995) Étude cytologique des formations fongiques en stromas se développant à la surface des racines de plants truffiers. Bull Acad et Soc Lorraines des Sciences 34, 1, 27-44

Poitou N, Cassin C (1989) Interaction entre le cuivre et différents champignons ectomycorhiziens comestibles. In : Mushroom Science XII (II). Proceedings of the Twelth International Congress on the Science and Cultivation of Edible Fungi, Braunschweig, Germany, 565-575

Pradel L (1914) Manuel de trufficulture : guide pratique. Baillière, Paris, $136 \mathrm{p}$

Prud'homme R (1972) Analyse morphostructurale appliquée à l'Aquitaine occidentale et au golfe de Gascogne. Thèse d'État, Bordeaux-I, $365 p$

Sourzat P, Kulifaj M, Montant C (1993) Résultats techniques sur la trufficulture à partir d'expérimentations conduites dans le Lot entre 1985 et 1992 . LPA Cahors, Le Montat, $40 p$

Verlhac A, Giraud M, Leteinturier J (1990) La truffe : guide pratique. CTIFL, Paris, $108 \mathrm{p}$

Voltz $M(1986)$ Variabilité spatiale des propriétés physiques du sol en milieu alluvial. Essais de cartographie quantitative des paramètres hydrodynamiques. Thèse docteur-ingénieur, Ensa, Montpellier, $198 \mathrm{p}$ 